\title{
Updated Limits on the Ultra-High Energy (UHE) Neutrino Flux from the RICE Experiment
}

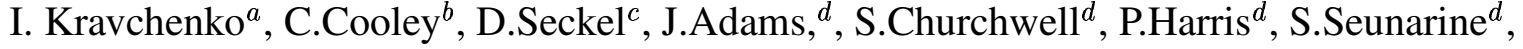

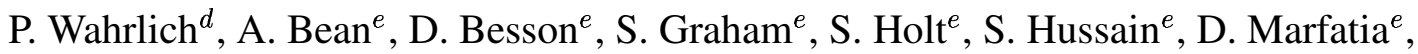

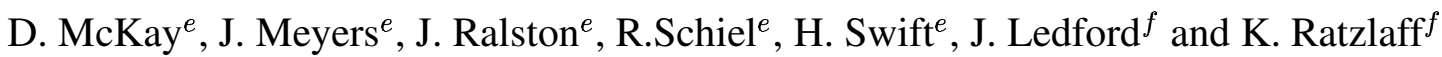 \\ (a) M.I.T Lab. for Nuclear Science, Cambridge, MA 02139 \\ (b) Whitman College Dept. of Physics, Walla Walla, WA 99362 \\ (c) Bartol Research Institute, U. of Delaware, Newark, DE 19716 \\ (d) Department of Physics and Astronomy, Private Bag 4800, U. of Canterbury, Christchurch, New Zealand \\ (e) U. Kansas Dept. of Physics and Astronomy, Lawrence KS 66045-2151 \\ (f) U. Kansas Instrumentation Design Laboratory, Lawrence KS 66045-2151 \\ Presenter: I. Kravchenko (ikrav@fnal.gov), usa-hussain-S-abs1-og25-oral
}

The RICE experiment (Radio Ice Cherenkov Experiment) at South Pole consists of an array of dipole antennas designed to detect the coherent radio frequency radiation produced by neutrino-induced showers in the Antarctic ice. We report updated limits on the ultra-high energy neutrino flux, based on RICE data taken between 2000 and 2004. These limits also reflect improvements in Monte Carlo simulations and detector modeling.

\section{Introduction and Overview of RICE}

The RICE experiment $[1,2,3]$ is designed to detect UHE neutrinos[4] with energies above $\sim 10 \mathrm{PeV}$. Potential sources include any source of UHE cosmic rays[5,6], although the details of such models are uncertain. UHECRs are observed at energies above $10^{19.5} \mathrm{eV}$ and are guaranteed to produce so called "GZK" neutrinos[7] with EeV energies during propagation. Such neutrinos are probes of the evolution of UHECR sources and provide a floor prediction useful for baseline detector design.

Early results from RICE[2] were based on one month (333 hours live) of data analysed for the presence of electron neutrinos. Subsequent studies included the possibility of hadronic showers and expanded the data collection time to greater than two years[3]. Here we describe the result of an improved analysis performed on data collected over a five year time frame. To date the experiment has not detected neutrinos, but places interesting limits on models of neutrino production in the energy range of $100 \mathrm{PeV}-1 \mathrm{ZeV}$.

During its operation RICE has consisted of an array of 16-20 radio antennas deployed within a roughly $200 \mathrm{~m} \times 200 \mathrm{~m}$ footprint at depths of $100 \mathrm{~m}-300 \mathrm{~m}$ near South Pole. The array is designed to intercept the Cherenkov cone of coherent, radio-frequency radiation from an UHE shower produced by a cosmic ray neutrino interaction in the Antarctic icecap. After pickup by the antenna, the signal is amplified and transmitted by coax cable to the surface. In the surface DAQ the signals are filtered, amplified again, and split into two copies: one for triggering and one for digitizing and analysis of the pulse waveform.

Data acquisition is triggered by the arrival of 4 pulse hits within a 1.2 microsecond window. The pulses must exceed a common discriminator threshold $V_{d}$ which coarsely tracks the background noise level. The pattern of arrival times is used to form an on-line veto against noise sources located on the surface. Events which pass the veto are digitized and recorded for off-line analysis, a process designed to pass neutrino induced events while rejecting manmade RF pulses and thermal noise fluctuations in the ice. The analysis includes tests based on wave form quality, vertex location and the ability to reconstruct a Cherenkov radiation pattern based on signal amplitudes in the receiver channels. 


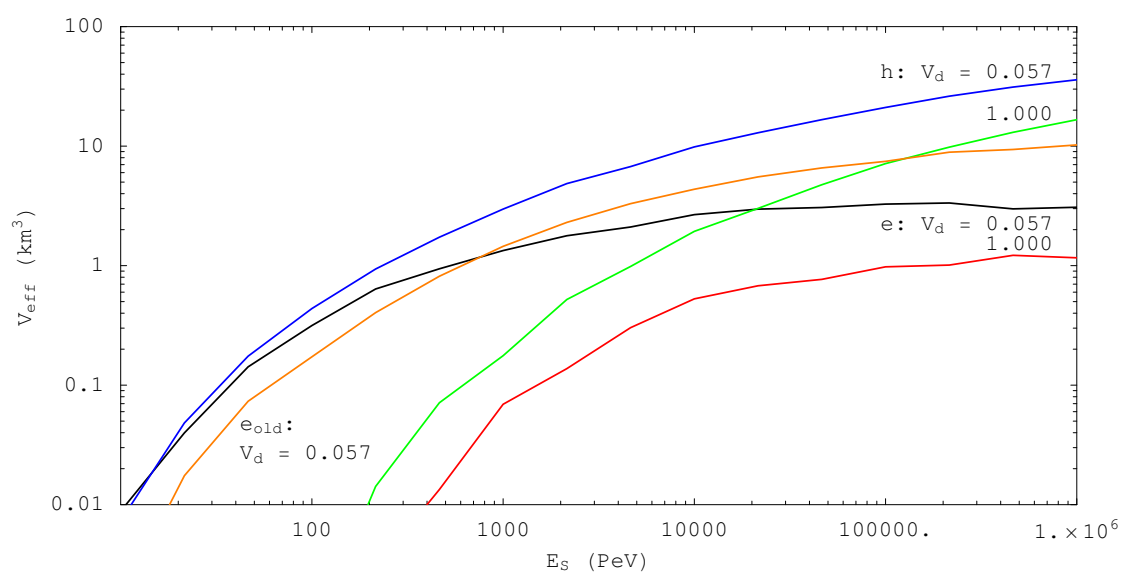

Figure 1. RICE effective volume $V_{\text {eff }}$ for electron $(e)$ and hadronic $(h)$ showers, shown at extreme values of the discriminator threshold $V_{d}$ used to trigger events. The fifth curve shows the previous result[3] for $e$-showers with $V_{d}=0.057 \mathrm{~V}$.

Effective Volume. Expectations for the RICE experiment are determined by Monte Carlo simulation. Details of the neutrino interaction determine the spectrum and radiation pattern of the shower. Showers initiated by electrons $(e)$ are elongated by the LPM effect, whereas hadronic $(h)$ showers initiated by quark jets are not. As a result electron initiated showers have narrow radiation patterns and exhibit reduced detection efficiency. We have refined the modeling of the showers and RF production, resulting in a modest increase in the signal strength[8]. Once produced, the radiation propagates to the antenna array, suffering both refraction and attenuation. Since the RICE antennas are located in the firn, a region of changing density and index of refraction[9], ray tracing calculations lead to reduction of the ice volume visible to the array. Recent in situ measurements[10] lead to a reduced attenuation length. These effects reduce $V_{\text {eff }}$ as compared to previous publications[1, 2, 3].

The final result of the Monte Carlo is an effective volume $V_{\text {eff }}$, defined as the volume integral of the detection efficiency for interaction vertices near the array. This integral is averaged over the solid angle of the neutrino beam, taken to be $2 \pi$ for a diffuse flux shadowed by the Earth. Figure 1 shows $V_{\text {eff }}$ for $e$ and $h$ showers at various discriminator thresholds as a function of energy. The new results are somewhat more sensitive at low energies due to adjustments for various instrument settings, but the improvements in modeling ice propagation reduce the sensitivity for $E_{s}>10^{20}$ by a factor of $\sim 3$ when compared to previous studies.

Online Veto and Analysis Efficiency. Monte Carlo events which would otherwise trigger the detector are used to estimate the efficiency for neutrino events to pass the online veto and the analysis filters. Modeled waveforms for a MC event are embedded in randomly selected "unbiased" events, collected to monitor noise characteristics. These events pass a code simulating the online veto with an efficiency of 0.86 . These events are also given to the analysis chain, and pass with an efficiency of 0.70. The combined efficiency is $\epsilon=0.60$.

Operations \& Results. During the five years covered in this analysis, RICE was operational approximately $80 \%$ of the time. For about half of this time, the South Pole Station satellite uplink operated at an amplitude which overwhelms the RICE DAQ. Additional deadtime arose while applying the online surface veto and readout of the oscilliscopes which comprise the digitization side of the DAQ, leaving a total of approximately 1.5 year of useful data. The bulk of this was acquired at thresholds of $0.2-0.4 \mathrm{~V}$, a level which may be compared to the curves in Figure. 1.

The final data set includes of order $10^{6}$ events that are analysed for neutrino interactions. After applying the 
analysis cuts, 43 events remained. These were examined by hand, and all were found to have defects that eliminated their consideration as neutrino events. Consequently, we can only report flux limits at this time.

\section{Limits on the flux of UHE neutrinos}

With no events, the RICE results may be used to place bounds on the number of events expected for a given model. Let the model have an overall normalization, $A$. If the expected number of events for $A=1$ is $N$, then with zero events the $95 \%$ CL upper limit on the normalization is $A_{95}=\frac{3}{N}$.

For a given flux model $\phi$, the number of events expected during the RICE exposure is given by

$$
N=\sum_{i, a, j} \int d t_{j} d E_{\nu} d y 2 \pi \epsilon V_{j}^{a}\left(E_{s}\left(E_{\nu}\right)\right) \frac{d \sigma_{i}^{a}}{d y}\left(E_{\nu}\right) \frac{d \phi_{i}}{d E_{\nu}}
$$

where $E_{\nu}$ is the neutrino energy, $\sigma$ is the $\nu N$ cross-section, $y$ is the inelasticity, $\epsilon$ is the combined efficiency, and $V_{j}$ is the effective volume for the experient configuration during time interval ${ }_{j}$. The event rate includes a sum over flavors $i$ and event types ${ }^{a}$. All flavors of neutrino create $h$-showers as recoil jets in charged (CC) and neutral (NC) current reactions. One flavor $\left(\nu_{e}\right)$ creates $e$-showers in CC events. For $h$-showers the shower energy is $E_{s}^{a}=y E_{\nu}$, whereas for the $e$-shower in $\nu_{e} \mathrm{CC}$ events $E_{s}^{a}=(1-y) E_{\nu}$.

For cross-sections we use isoscalar-target SM cross sections evolved to high energy. The ingredients include the tree-level parton amplitudes and CTEQ 6.2 parton distribution functions, with $\mathrm{Q}^{2}$ extrapolation where required. We include a $20 \%$ reduction due to the nuclear effects in oxygen. For a given flux model, neutrino mixing is assumed to distribute the total flux equally across all three flavors. Due to the competition between the LPM effect and an average inelasticity of $y \sim 0.2$, for $E_{\nu}<1 \mathrm{EeV}$ detection of an isoflavor flux is dominated by $e$-showers, but $h$-showers dominate above $1 \mathrm{EeV}$.

Our $95 \%$ C.L. bounds on representative $\nu$-flux models are shown in Figure. 2. The illustrative AGN models are ruled out at $95 \%$ C.L., but the Waxmann-Bahcall standard is below our limits. The GZK flux models differ substantially. ESS and PJ, keyed to models of the star formation rate, are below the RICE sensitivity. The KKSS flux, constructed to saturate bounds derived from EGRET observations, is just barely consistent with our $95 \%$ C.L. limit, i.e. RICE should have detected 2 events for this model but saw none. Direct UHE neutrino detection is competitive with limits derived from complementary photon and cosmic ray observations.

\section{Summary and outlook}

With improvements in the simulation of neutrino induced showers and the propagation of pulses from showers to antennas, we have analyzed new RICE data from 2003-2004 and re-analyzed data from 2000-2002. The analysis finds no $\nu$ 's. The net result is a new set of bounds on cosmogenic neutrino fluxes that are somewhat tighter than our ICRC 2003 proceedings [3]. A more detailed description of the new results is in preparation.

The current RICE array is expected to take data for the next 2 - 3 years, with corresponding improvements in sensitivity. We are developing a successor to the current RICE array that features analog signal transmission by optical fiber coupled to surface digitizer boards to significantly improve the bandwidth of the experiment, and antenna clusters with local hardware coincidence to veto surface noise and lower the threshold for true neutrino signals. The above improvements are progressing in tandem with studies of an expanded radio array, which in combination with an acoustic detector could be operated as part of the IceCube observatory[11]. 


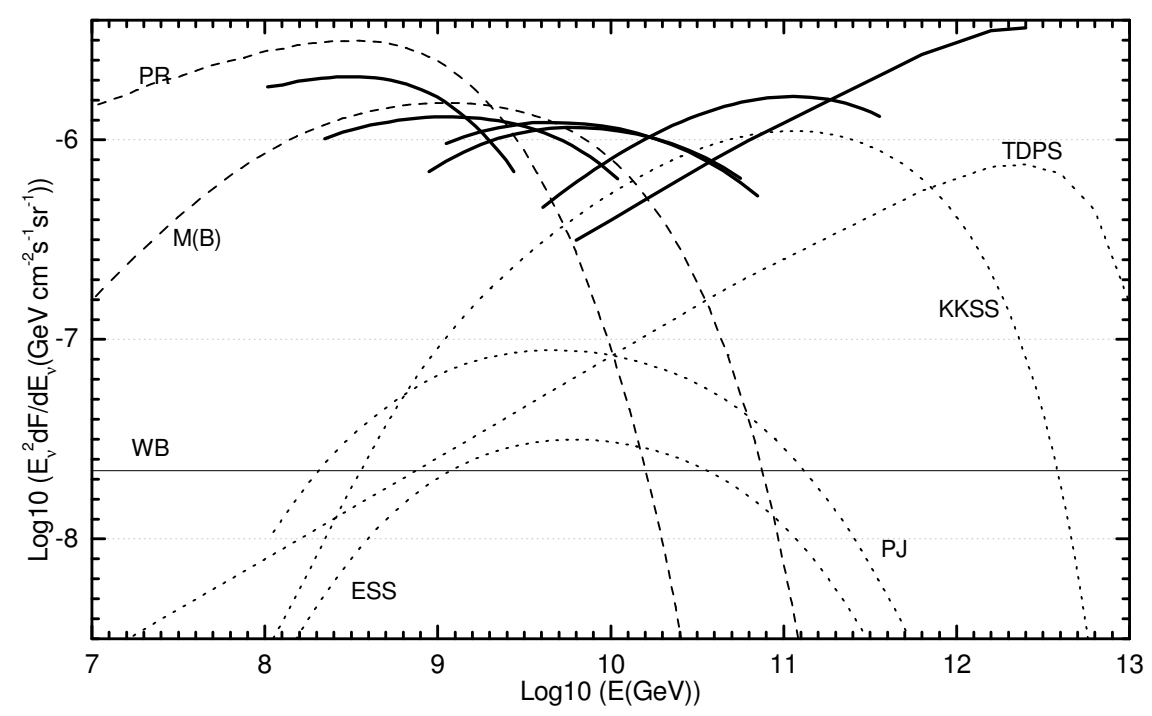

Figure 2. Upper bounds on total (all flavor) neutrino fluxes for AGN models of PR and MB [5], GZK neutrino models of ESS, PJ, and KKSS [7], and the topological defect model of PS, due to all flavor NC+CC interactions, based on 2000-2004 RICE livetime of about $13200 \mathrm{hrs}$. Thin curves are for model fluxes and the thick curves are the corresponding bounds. The energy range covered by a bound represents the central $80 \%$ of the event rate.

\section{Acknowledgements}

We acknowledge the NSF Office of Polar Programs for support. The RICE experiment would not have been possible without the generous logistical and material support of the AMANDA collaboration. We thank P. Broughton, M. Offenbacher, and C. Hammock for assistance operating the experiment.

\section{References}

[1] RICE Collaboration, I. Kravchenko et al., Astroparticle Physics 19, 15 (2003).

[2] RICE Collaboration, I. Kravchenko et al., Astroparticle Physics, 20, 195 (2003).

[3] RICE collaboration, I. Kravchenko et. al, in proceedings of 28th ICRC, Tokyo, 2003 (Universal Academy Press, Tokyo, 2003).

[4] V.S. Berezinsky and G.T. Zatsepin, Phys. Lett. 28B, 423 (1969); F.W. Stecker, Astrophys. J. 228, 919 (1979)

[5] R. J. Protheroe, astro-ph/9607165; K. Mannheim, Astropart. Phys. 3, 295 (1995).

[6] R. J. Protheroe and T. Stanev, Phys.Rev.Lett. 77 (1996) 3708-3711; Erratum-ibid. 78 (1997) 3420.

[7] R. Protheroe and P. Johnson, astro-ph/9506119; R. Engel, D. Seckel and T. Stanev, Phys. ReV. D 64, 093010 (2001); O. Kalashev et al., Phys. Rev. D 66, 063004 (2002).

[8] S. Hussain and D. McKay, Phys. Rev. D 70, 103003 (2004); S. Hussain and D. Seckel, in preparation.

[9] Kravchenko, I, et al. "In situ measurements of the index of refraction of the South Polar firn with the RICE detector", J. Glaciol. in press.

[10] S. W. Barwick et al., submitted to J. Glaciol (2004).

[11] J. Vandenbroucke, Proceedings of 29th ICRC (2005). 